\title{
Reliable Wireless Sensor Protocol Based Health Monitoring System
}

\author{
Munther Naif Thiyab ${ }^{1}$ \\ ${ }^{1}$ Engineering College, University of Anbar, Anbar, Iraq \\ Correspondence: Engineering College, University of Anbar, Anbar, Iraq. Tel: 00-964-782-490-0180. E-mail: \\ abunife2002@yahoo.com
}

Received: February 23, 2016

Accepted: April 20, 2016

Online Published: June 7, 2016

doi:10.5539/mas.v10n8p56

URL: http://dx.doi.org/10.5539/mas.v10n8p56

The research is financed by (Sponsoring information).

\begin{abstract}
The patient monitoring during normal activity is getting more and more significant as a standard method to prevent craniology process for detection of transient ischemiaperiod , cardiac arrhythmias as well as silent myocardium ischemic. In this paper, we mainly focus on the structureand the composition of awireless $(600 \mathrm{~m})$ synchronizationarrhythmias invigilator as well as a alarming device for patients. To realize the corresponding functions, a Wireless Transducer Protocol (WTP) based on Time Divisibility Double (TDD) is what we require, the implemented system is totally composed of four main nodes includingthe Core (dominant) node which is a personal computer-based Graphic user system. It works at anstable frequency of $915 \mathrm{MHz}$. The passive node is a digital signal processor-based board connected with the patients whichcould receive two channels of full-spectrum ECG signal at the same time, and uploads processed data at a specificperiod to the core node. The MPC8260 communications and the computeris composed of the dominant nodedesigned to receive data, decompress, and analyze the latest data baggage. The SRWF-501F915 completednoderealize the data communication.
\end{abstract}

Keywords: health monitoring, arrhythmias, data acquisition, wireless monitoring

\section{Introduction}

It is favorable for medical practitioners to get an appropriate and adequate treat if diagnosing and continuous monitoring of cardiacarrhythmias from time to time could be effective;meanwhile, it is alsoimportant for health care providers to succeed in the controlling of the disease ((Hussain, Kamarul \& Ahmed, 2013; Hussain, Kamarul, Mithaq \& Ahmed, 2015) (Yen P. C. et. al., 2015). Accordingly, the demand for Distance real-time as well asconstant monitoring of cardiac arrhythmias could last forever.

Despite the fact thatnumerous diagnosis technologies adoptalikemeans to surveillancecountless physical data, for example, "Convenient Heart Invigilator" (Ahmed, Nubli, Siong, Khairi \& Noraziah, 2010), "Wireless ECG" (Bhavik, 2002), Long-distanceInvigilator System (Fakharuddin, Ahmed, Abdullah, \& Rauf, 2010), Wireless ECG Invigilator (Haiying, Kun, Jean \& Laurent, 2005), Continuing Metrazol Exploration System (Shah, Der, Ruggerio, Heidenreich, Massie, 1998), but actually, none of thosecould actually avoid thetraditional disadvantages such asline off procedure, largeenergy consumption and cost yetnear distance, single invigilator as well as always accompanied with an expensive backbones includingweb lines and GPS (Rauf, Ahmed, Ghazali, Fakharuddin, 2010) .

Fortunately, the system introduced in this articlegather the functions to detect, classify, display, compress, store, and even upload the arrhythmias data samples, meanwhile, it can also enable the patients and the physician to communicate by the means of short messages, beside,different the one before ,the equipment first start using an developed lower cost design and energy consumption but with a more specific methodologies. And because of the complication of the system; we have adopted some reliable technologies such as a WSP and constructing nodes design methods to ensure that the system could be separate into different nodes.

\section{Theoretical Background}

The system mainly includes four nodes and the central node includes several building blocks as in Fig. 1 . 


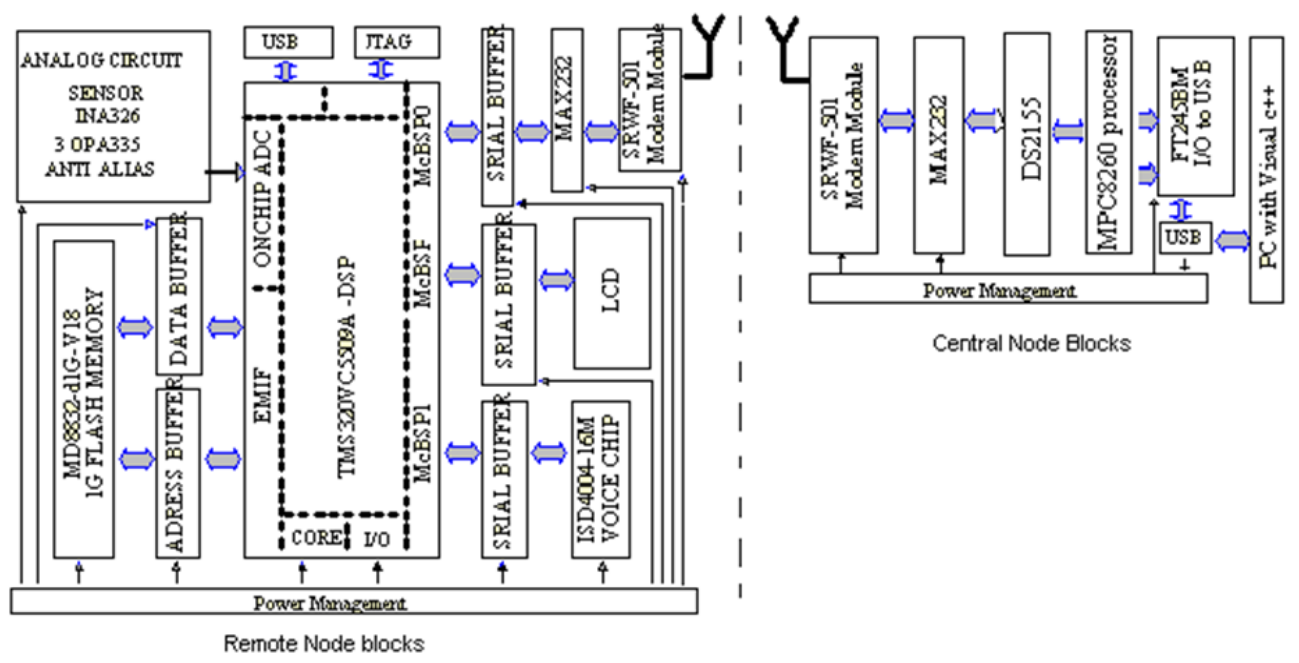

Figure 1. System Block Diagram

\subsection{The Communication Link}

For setting up the communication link a Wireless Sensor Protocol (WSP) was introduced, where use TDD (Time Division Duplex) plan to send data periodically to hub node. By using three-layer little network stack as shown in Figure 2, the WSP operates with APP (Application layer) related to the obtainment of ECG data pre-processing via DSP processor as well as analog circuit, also APP is concerned with development of User Application Program to sample, compress, decompress construct, display and analyze the ECG signal on PC, the Medium Access Control Layer (MAC) though the MPC8260 and DSP processorsis in charge of safeguarding the network consisting of the establishment of link, altering the transmission of modes data, supporting the whole types of network packet and the address of node, the management of network and data acquisition's transformation, which consists of the interface to the APP, in which the synchronization of sample data is required during the sending process, so as the interface to the PHY, the PHY (Physical layer) needs an interface to the module of SRWF-501F915 RF and the layer of MAC, besides, the PHY is in charge of acquiring 'dependable' correspondence between different nodes.Namely, at each node the received data transferred to the Layer of MAC has no errer, which will be performed, the PHY will carry out; CRC and DW (Data Whitening) for the minimization of packet's DC bias as well as the provision of error detection. The WSP specifies different packet types include: Connect Request, Disconnect Request, Acknowledge/poll, Request Settings and Data Transfer (Ahmed, Rauf, Fakharuddin, \&Yao, 2010, Ahmed, Hawari, \& akharuddin, 2010).

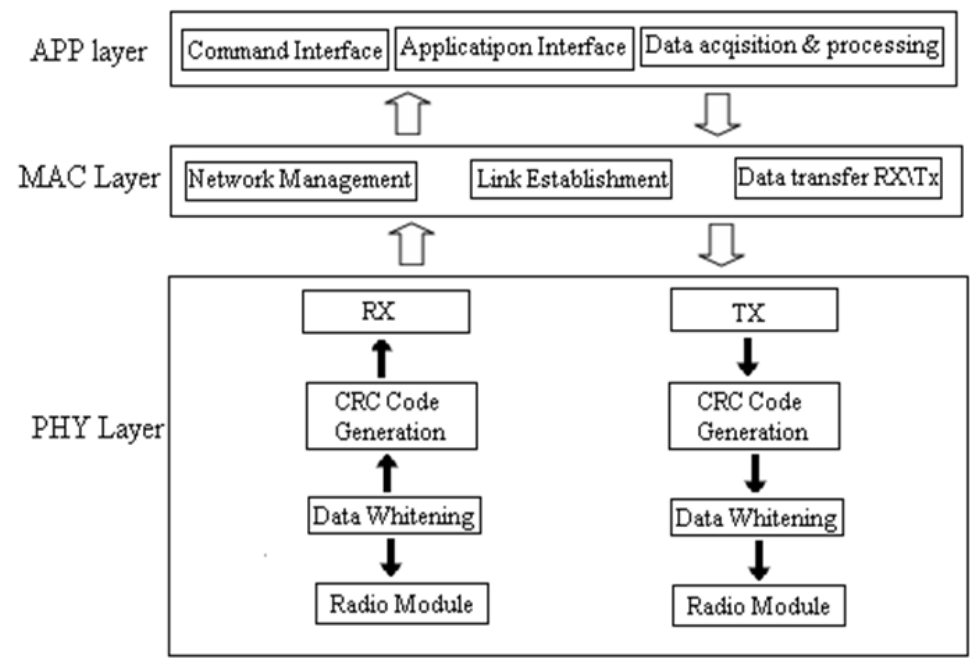

Figure 2. WSP Architecture 


\subsection{The WSP Mechanism}

The system's first startup is carried out in master node's APP layer.Visual C++ creates GUI to give tips to the user by the initialization of the wireless connection. In turn, it will send data through a USB port to the communication processor of MPC8260, which consists of PHY as well as MAC layers.A packet is transferred on the PHY layer, which consists of information on the management of network.The message's decoding and the transmission packets creation are performed prior to the final transfer of data via the transceiver (Kumar, Bansal Tiwari, Nayak \& Narayanan, 2014; Shun, Chih, Lin \& Shuenn, 2014).

The packet is received by the transceiver at remote nodes and transferred in the processor of DSP.The message is decoded in the layer of PHY, followed by passing the information on the layer of MAC. Firstly, the layer of MAC examines to decide whether the packet is valid derived from the central node, if this is the case, it reinstalls the clock and achieves the slot time of transmission.Then, it transfers a query on the layer of APP, and starts the ECG data sampling and storage in the flash memory of DSP processor.

Before the transmission, the layer of MAC alters the transceiver mode to transmit mode.Afterwards, the layer of MAC makes an extraction of ECG data sample, and transfers it on the layer of PHY.Again, such information is created and formatted in a packet, which will be transfer back through the central node.

In the end, after receiving the data, it transfers it once more via the special layers till arriving at the layer of PHY.When the layer of PHY has accepted large amounts of data from remote node within the network, it is capable of the creation of signal of ECG on PC.

The size of time slots is dependent on the symbol rate of the RFSRWF-501F915 module as well as the time required for the RF SRWF-501F915 to switch between receiving and transmitting modes.

\section{Proposed Protocol}

\subsection{Remote Nodes Algorithms}

As mentioned before, the Node is composed of two kinds of operating including Stand-Alone and Slave-Master. Hence it has individual system for each one for which both modes has a demand for basic support functions of certain common, and thus the software has a modular structure. Then the support routines are able to be accepted into any systems as a normal operational methodto achieve minimum energy consumption. The whole algorithms are based on TI digital signal processor on-chip algorithm.

As what is shown in the Figure three, it demonstrates the TMS320VC5509A-digital signal processor algorithm's flow chart.The data was generated in Data Generate Studio v. 3.1. Upon power-up, the digital signal processor chip is derived from instant memory assystem theliquid crystal display; after the user give out the mode (if stand-alone); a graphic user interface window is supposed to show up whichenables the subscribers to select a accordance function and the digital signal processor chip operates the selected supporting (receive ECG signal, ADC converse, exam. Arrhythmias, showing essential ECG signal, generating detecting result, stamping, giving out alarming or recording things. At the same time, the processor chip of digital processor is responsible for the ancillary equipment to achieve the appropriate operations.Besides, if the mode is master-slave; the chip of DSP obtains the signal, make conversion of it into ADC, filters it, compress it and stores it for waiting the central note to establish a new transmission link in its specific time slot. Ifso the DSP chip decompresses and passes the data to the SRWF-501F915 modem for transmitting. 


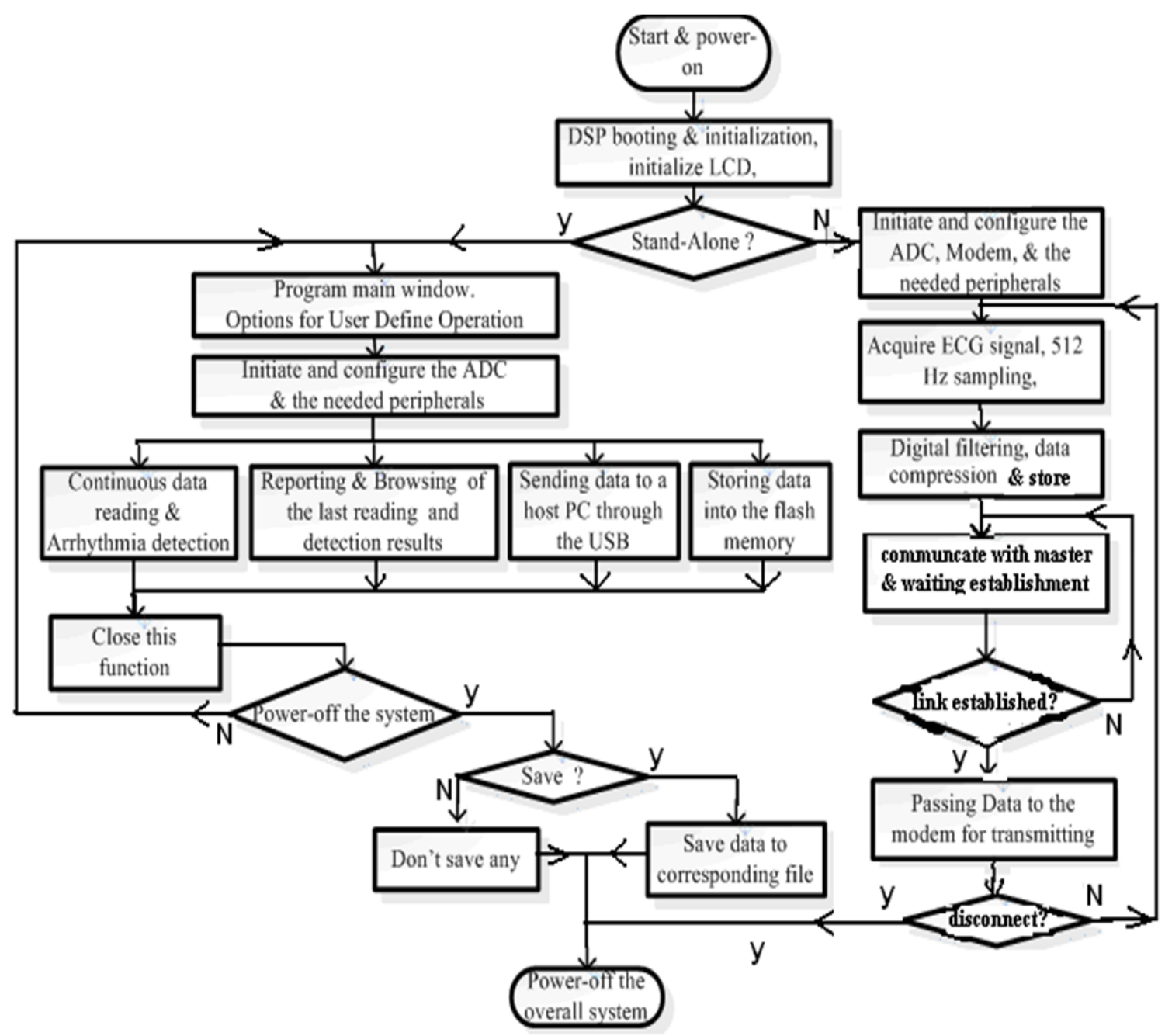

Figure 3. Remote nodes algorithms

\subsection{Central Node Algorithms}

These algorithms are composed of two nodes, one is based on MPC8260 algorithms and the other one is based on the personal computer application. Figure four demonstrates the program procedure.Upon power-op the algorithm loads, initiates the processor I/O and TDM ports, sets the modemto the active mode, configure the time slots register, initializethe connection and synchronization period, assigning each (online) remote node with a particular time slot. Changes into receive mode once the entire packet has been sent. Once the synchronization is accomplished, it receives data from the particular remote node at its given time slot, and sends the data to the PC This cycle will be repeated certain number off times before Central will eitherresynchronize or disconnect. Figure 5 shows the structure of the application program based on personal computer; Art tools nowadays arewidely adopted in the computer system using Visual $\mathrm{C}++$. The program is used to acquire, analyze, construct and display the patients ECGs periodically. 


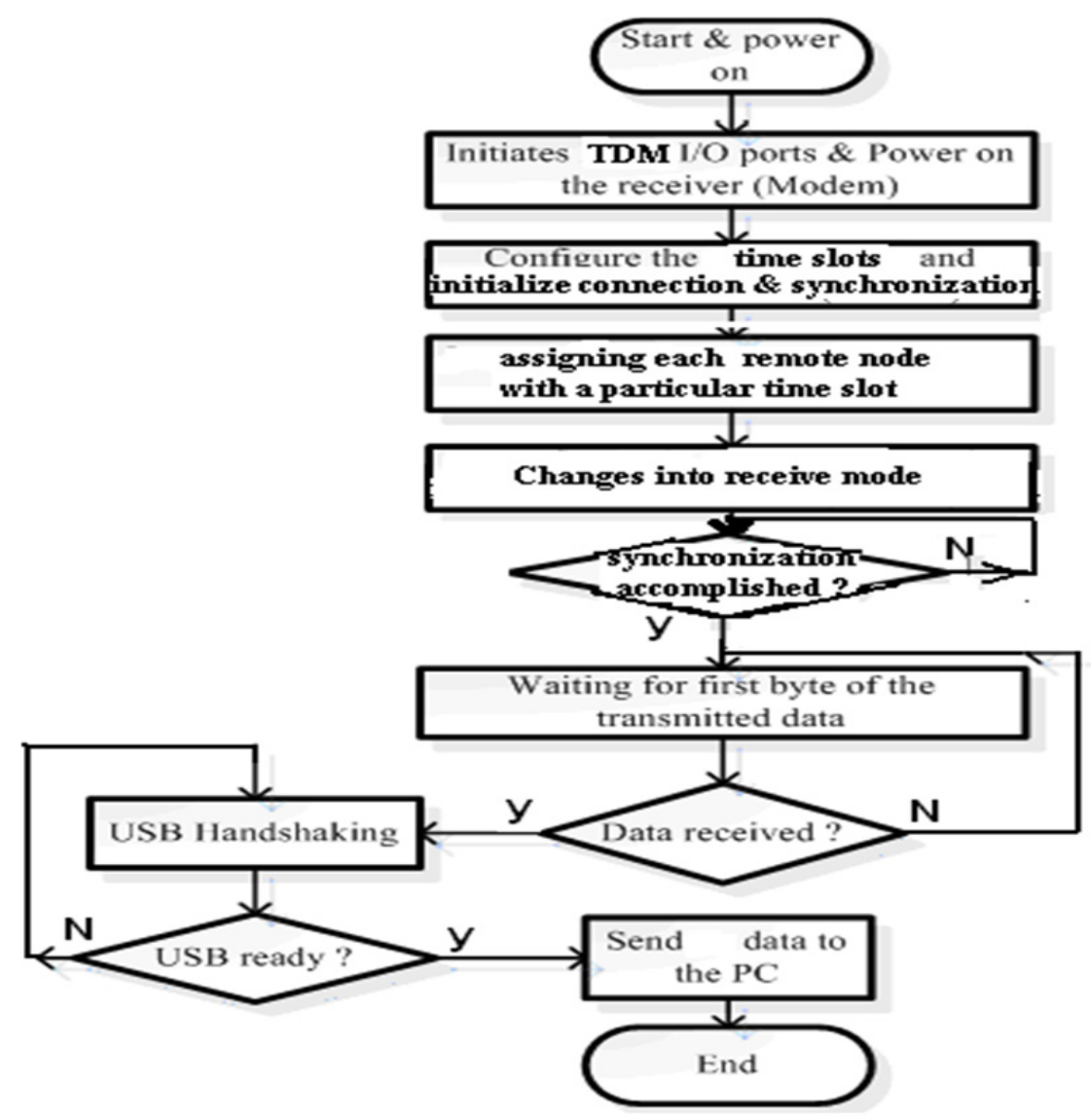

Figure 4. MPC8260 processor -based Algorithm flowchart

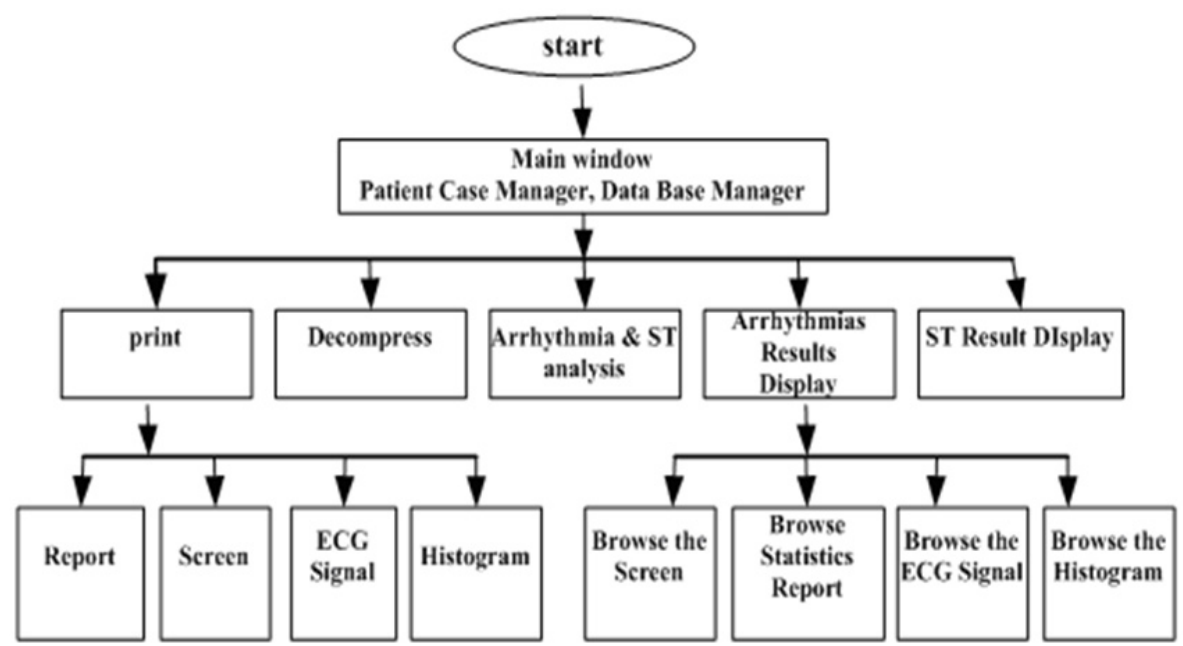

Figure 5. PC-based System architecture

\section{Results And Discussion}

By the WSP architecture implementation experiments, comparatively accurate results are realized if every 6 cycles the re-synchronization was performed for the network.Nevertheless, by including more nodes, it nearly will be sure to lead to more re-synchronization demand than fixed periods. As what we can see in table 1, System consequenceagainst artificial outcomes of Arrhythmia Detecting and ECG wave values for fourpatients is clearly showed in the figure clearly. Fig. 6 shows an ECG wave form for an old patient, who suffers cardiac disorder. 
Table 1. System-Based versus Manual, Arrhythmias Detection Parameters Results

\begin{tabular}{lll}
\hline No & Part & Power (mA) \\
\hline 1 & SRWF-501F915 modem & 40.00 \\
2 & ISD4004 voice & 15.00 \\
3 & ECG front-end analog circuitry & 03.10 \\
4 & TMS320VC5509A DSP \& it's & 46.00 \\
& Peripherals & \\
& Total & 104.10 \\
\hline
\end{tabular}

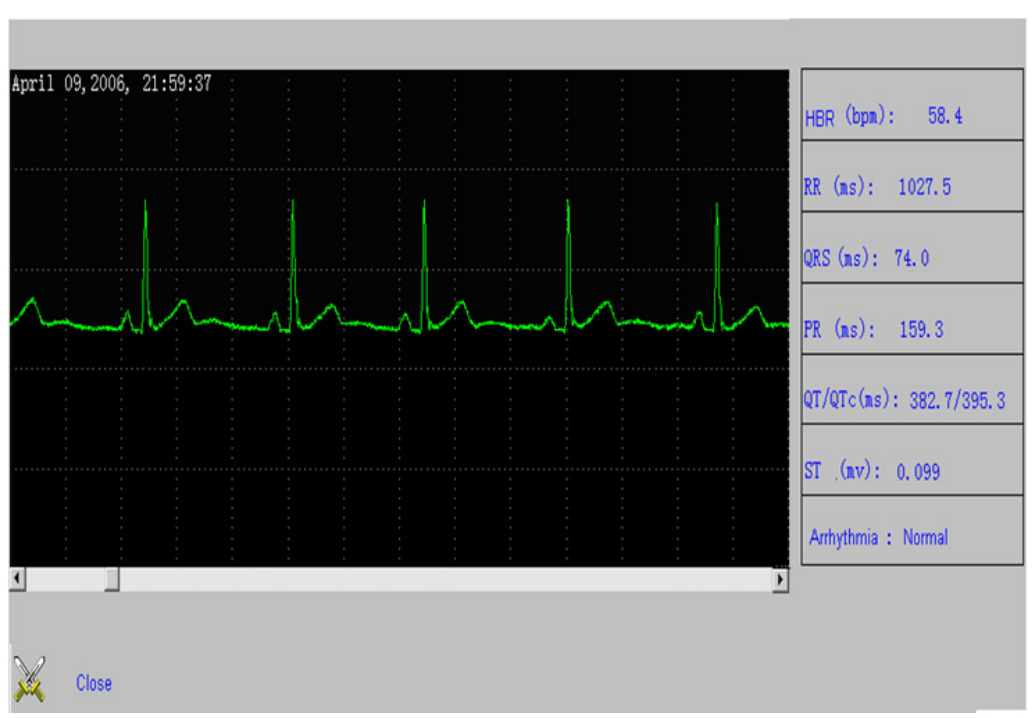

Figure 6. Arrhythmias\& ECG wave Result window

Power consumption characters our design tremendously for the total energy consumption is always denoted as the current which is provided to the whole inputs during the regular operation of the system. In order to achieve our aim of lowpower consumption, advanced technologies have been followed and considered, for example ,Sleeping Modes, Clock Frequency Controlling, Controlling over raw Peripherals, Controlling over raw Outputs, unique Program Techniques;Table 2 reflectstherules of total energy's dissipationthrough the Long-distance Nodes.

Table 2. Remote Node Power Dissipation (operating)

\begin{tabular}{|c|c|c|c|c|c|c|}
\hline $\begin{array}{l}\text { Patient } \\
\text { No }\end{array}$ & $\begin{array}{l}\text { Results } \\
\text { Method }\end{array}$ & $\underline{\underline{y}}$ & 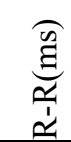 & $\begin{array}{l}\text { कू } \\
\text { है } \\
\text { है }\end{array}$ & 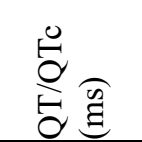 & 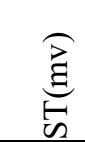 \\
\hline \multirow{2}{*}{1} & System-based & 64 & 165 & 85 & $393 / 392$ & 0.034 \\
\hline & Manual & 62 & 161 & 83 & $394 / 393$ & 0.042 \\
\hline \multirow{2}{*}{2} & System-based & 75 & 140 & 78 & $368 / 374$ & 0.025 \\
\hline & Manual & 72 & 146 & 76 & $370 / 372$ & 0.031 \\
\hline \multirow{2}{*}{3} & System-based & 82 & 168 & 83 & $351 / 369$ & 0.017 \\
\hline & Manual & 81 & 164 & 79 & $359 / 368$ & 0.022 \\
\hline \multirow{2}{*}{4} & System-based & 61 & 128 & 73 & $374 / 381$ & 0.062 \\
\hline & Manual & 62 & 130 & 72 & $375 / 384$ & 0.079 \\
\hline
\end{tabular}




\section{Conclusion}

The system now offers numerous patients including real time, low energy consuming, long distance, low cost, and Dual-modes Arrhythmias Invigilator.it is even possible for poor people in the developing countries to enjoy the technology even if there are no mobile lines, web net -based systems, or GPS. Use MPC8260 and TMS320vVC5509A-DSP processor of communications for the construction of blocks for the system of monitoring, which possesses the compact size, intelligence and reliability's benefits. The advance integrated chips, external component, the minimization of wirings is achieved.It describes and introduces a wireless sensor protocol that is reliable, besides, in order to develop such DSP-based system; a processing technique of powerful digital signal was used.The system`s further benefit is the consumption that has low power and is attractive for applications that are portable.

\section{References}

Ahmed, N. A., Nubli, M., Siong. T. C., Khairi, F., \& Noraziah, A. (2010). Enhancement of real-time multi-patient monitoring system based on wireless sensor networks. International Journal of Physical Sciences, 6(4), 664-670. http://dx.doi.org/10.5897/JJPS10.611

Ahmed, N. A., Rauf, M., Fakharuddin A., \& Yao X. (2011). Public transport monitoring with route and dispatch management system. African Journal of Business Management,5(22), 9106.

Bhavik, K. K. (2002). Wireless ECG. Thesis University of Queensland, School of information Technology and electrical Engineering, 2002.

Fakharuddin, A., Ahmed, N. A., Abdullah, R., Ahmad, M. R. B., \& Rauf, M. (2010). Modern control of electrical consumption system using wireless, reader designed algorithm. International Conference Information Networking and Automation, pp. V1-184-V1-187. http://dx.doi.org/10.1109/ICINA.2010.5636408.

Haiying, Z., Kun, M. H., Jean, P., \& Laurent, G. (2005). Real-Time Continuous Cardiac Arrhythmias Detection System (RECAD). 27th Annual Conference IEEE, Engineering in Medicine and Biology. http://dx.doi.org/10.1109/IEMBS.2005.1616555.

Hussain, K. K. , Kamarul, H. G., \& Ahmed, N. A., (2013). Features Extraction Technique For Ecg Recording Paper. International Conference On Artificial Intelligence In Computer Science, pp. 243-248. e-ISBN 978-967-11768-3-2.

Hussain, K. K., Kamarul, H. G., Mithaq, N. R., \& Ahmed, N. A. (2015). A New Approach in a Gray-Level Image Contrast Enhancement by using Fuzzy Logic Technique. International Journal of Scientific Research in Science, Engineering and Technology, 1(1), 19-23. ISSN : 2395-1990.

Kumar, S., Bansal, A., Tiwari, V. N., Nayak, M. M., \& Narayanan, R. V. (2014). Remote health monitoring system for detecting cardiac disorders. IEEE International Conference on Bioinformatics and Biomedicine (BIBM), pp. 30-34. http://dx.doi.org/10.1109/BIBM.2014.6999264

Rauf, M., Ahmed, N. A., Ghazali, K. H. B., \& Fakharuddin, A. (2010). Intelligent multi ID buses navigation system with efficient data transmission technique. International Conference Information Networking and Automation (ICINA), pp. V1-57-V1-61. http://dx.doi.org/10.1109/ICINA.2010.5636434

Shah, N. B., Der, E., Ruggerio, C., Heidenreich, P. A., \& Massie, B. M. (1998). Prevention of hospitalizations for heart failure with an interactive home monitoring program. American Heart Journal, 135(3), 373-380. PMID: 9506321

Shun, R. S., Chih, C. H., Lin, M. P. H., \& Shuenn, Y. L. (2014). A novel approach for ECG data compression in healthcare monitoring system. IEEE International Symposium on Bioelectronics and Bioinformatics, pp. 1-4. http://dx.doi.org/10.1109/ISBB.2014.6820946

Yen, P. C., et. al., (2015). An Injectable 64 ECG Mixed-Signal SoC in $65 \mathrm{~nm}$ for Arrhythmia Monitoring. IEEE Journal of Solid-State Circuits, 50(1), 375-390. http://dx.doi.org/10.1109/JSSC.2014.2364036

\section{Copyrights}

Copyright for this article is retained by the author(s), with first publication rights granted to the journal.

This is an open-access article distributed under the terms and conditions of the Creative Commons Attribution license (http://creativecommons.org/licenses/by/3.0/). 